\title{
Design and Analysis of an Optimized Formula 3 Nosecone Structure
}

\author{
ARCHIT DESHPANDE, NAVEEN VENUGOPAL \\ and HAMID DALIR
}

\begin{abstract}
In order to ensure the driver safety in motorsport crashes, special crash structures are designed to absorb the race car's kinetic energy and limit the decelerations acting on the human body. The use of Carbon fibre epoxy as a primary structural material has been evident in the motorsport industry. By utilizing monolithic structure for crash, large amount of energy can be absorbed. However, the energy absorbing capacity, unlike metals, is highly dependent on the geometry, number of layups and layup orientation angles. By optimizing the plies and the orientation along the geometric cross-section, the deceleration of the vehicle can be controlled. For the FIA crash test regulations, the deceleration was limited to $5 \mathrm{~g}$ 's for the first $150 \mathrm{~mm}$ of crushing and the average deceleration was limited to $25 \mathrm{~g}$ 's. By dividing the geometry into sections, the ply orientation, and number of plies were varied. This resulted in a nosecone structure weighing around $2.1 \mathrm{kgs}$, but able to meet the above requirements. From the research ${ }^{1}$ it is evident that the Specific Energy Absorption (SEA) is not only a function of geometric cross-section $(\varphi)$ but also the angle of attack $(\beta)$. The angles of attack were varied from $5.5^{\circ}$ to $32.5^{\circ}$ and the effects on SEA were observed. The dynamic simulations were conducted in explicit solver LS-DYNA using Mat_ENHANCED_COMPOSITE_DAMAGE material model (MAT54). The simulation results were validated with crush test data for energy absorbed.
\end{abstract}

\footnotetext{
${ }^{1}$ Archit M. Deshpande, Naveen Venugopal, Hamid Dalir, Advanced Composite Structures Engineering Laboratory (ACSEL), Purdue School of Engineering and Technology, IUPUI, 799 W Michigan St, Indianapolis, Indiana, 46202, U.S.A
}

This is the author's manuscript of the work published in final edited form as:

Deshpande, A., Venugopal, N., \& Dalir, H. (2019). Design and Analysis of an Optimized Formula 3 Nosecone Structure. Proceedings of the American Society for Composites - Thirty-Fourth Technical Conference. https:// doi.org/10.12783/asc34/31257 


\section{INTRODUCTION}

Vehicles travelling at a high speed is always has a risk of safety of its occupants associated with it. This cannot be more evident than racing in the motorsports industry. Over the years, it has been observed that the leading cause of severe injuries and fatalities is concussion to head and neck. As suggested in the research conducted by Weaver et. Al [1], there is a direct relation between g-forces of deceleration and head injury probability. The research analyses crashes in Indy Racing League (IRL) from 1996 to 2003 with a data size of 374 crashes, divided into two sections. One with impact $>50 \mathrm{~g}$ and one with lesser forces. A crash with $\mathrm{g}>50$ resulted in $16 \%$ probability of traumatic brain injuries. This research suggests that a controlled g-force is necessary for the safety of driver.

In order to regulate the $\mathrm{g}$-forces acting on a driver, a sacrificial crash structure is designed and mandated in every vehicle. The material of this structure plays an important role in determining the peak and mean g-forces acting on the driver. Previously, Aluminium and high strength foams were common in the motorsport industry. In 1981, carbon fibre was utilized in the chassis development of McLaren MP4/1. Carbon fibre has very specific strength and stiffnesses. It also has very high Specific Energy Absorption due to the difference in the failure mechanism in carbon fibre over the conventional metal. Metals being ductile in nature absorb energy by plastic deformation while carbon fibre absorbs energy by splaying and fragmentation. In an event of crash, the sacrificial structure fragments into fine fragmented particles which makes it less harmful for the driver or other vehicles, thereby absorbing energy of impact. This makes CFRP an ideal material to be utilized in the crash structure.

For the safety of driver and other stakeholders, FIA (Fédération Internationale de l'Automobile) [2] has established certain rules for each category of motorsports. Different vehicles have different performance levels and thus it is necessary for the governing body to regulate the sport appropriately. FIA oversees the vehicle structural safety including the monocoque and crash structure design apart from other components like the road safety, runoff areas and crash barriers. As the vehicle in development has speeds comparable to Formula 3, the impact structure designed here is as per the Formula 3 rules. Furthermore, the rules ensure the driver is enclosed in a survival cell with sacrificial crash structures at front, side and rear of the cell. FIA has limited the peak and average g-forces, acting on the driver, with respect to crush front propagation in all directions. The survival cell is also subjected to multiple static and intrusion tests. With the introduction of these regulations, the fatalities and injuries occurring in the motorsports industry has reduced significantly.

The crash absorbing mechanism of carbon fibre composites unlike metals, cannot be predicted accurately because of its complex failure mechanisms. As suggested by Hull [3], the failure of composites can occur in eight different ways: fracture in tension, compression, shear parallel in both parallel and perpendicular directions of fibre, interlaminar fractures in tension and shear, and matrix shear. The failure during crash can be either one or a mixture of all the failures, thus 
leading to large uncertainty in prediction of results. Thus, it is common in the design process to validate all results experimentally. Hull [3] was able to generalize two modes of failures 'splaying' and 'fragmentation'. The crushing mechanism is a combination of these and is highly dependent on the geometry of the crush piece. Farley and Jones [4], Carruthers [5] also identified these as the failure modes and came to similar conclusions. It is common in all research; the failure method is geometry dependent and very difficult to simplify and quantify.

Although it is not yet possible to generalize the physical crushing mechanism, research has been conducted to connect experimental results with theoretical design phase. Wade [6] was able to link SEA with geometry hence quantifying the energy absorbing mechanisms with geometry. A dimensionless parameter $\varphi$ was defined which denoted the ratio of curvature in the cross-sectional perimeter. The value of SEA as a function of $\varphi$ was plotted. By utilizing the given data, any crash box can be designed for the given material provided there is no angle of attack.

In the research conducted by Heimbs [7] et. al, the nose cone of a Formula 1 car is divided into various sections. Each section has varying ply layup, ply angle and ply thickness. The research showed promising results with MAT54 material model in LS-DYNA. As the nose cone for the Prototype-2 car resembles a formula type vehicle, utilizing similar simulation techniques would provide positive correlation between theoretical and experimental results.

The aim of this study is to apply the finite element codes and predict the crash g-forces using Wade [6] dimensionless parameter $\varphi$. Furthermore, a parameter $\beta$ is defined which represents the angle of attack of each section. This angle of attack would be plotted against the observed SEA. This study would however consider only straight frontal impact with a solid rigid wall with no deviations in the angle of impact. The focus of this research is to model composite material crushing and help quantify the experimental results with design parameters.

\section{OBJECTIVE}

According to the rules of FIA Formula 3, the frontal impact test needs to be carried out at a speed of $14 \mathrm{~m} / \mathrm{sec}$ with a test sled weighing $600 \mathrm{kgs}$. The rules specify the deformable part to decelerate at a maximum of $5 \mathrm{~g}$ 's for the first $150 \mathrm{~mm}$ and an overall average $\mathrm{g}$-force to be lower than $25 \mathrm{~g}$ including the first $5 \mathrm{~g}$ limit, Fig (1). Furthermore, the entire velocity of the vehicle needs to be dissipated without the failure of any other component of the survival cell. Also, a nose push-off test is carried out to regulate the failure of nosecone for an angle impact. This test is out of scope for this research, as it more aligns with static analysis of component. 


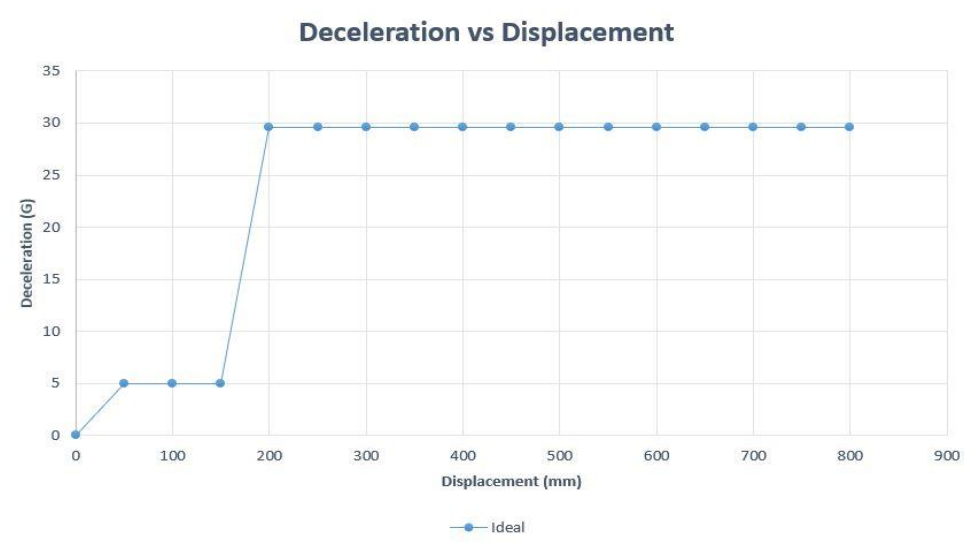

Figure 1. Ideal deceleration graph for a Formula 3 nosecone.

\section{METHODOLOGY}

As dynamic simulations require large computational resources and time, the verification of material properties and simulation setup is carried out on simple geometries. Various CAD geometries were meshed and simulated. The results were later compared against experimental tests for adjusting certain material parameters to reduce the simulation errors. The simulation time for these simple geometries were observed to vary within 8 minutes.

The finalized parameters were then utilized for the actual meshed CAD file. After running through various optimization techniques, a finalized model was generated using Quality Index based tool in HyperMesh. This model was then compared against standard industrial designs. The advantages and disadvantages of the generated results were discussed.

\section{FINITE ELEMENT MODELLING}

\section{Material Properties}

The material of construction for the monolithic design of the nose cone was selected as T700/2510 carbon fibre epoxy prepreg which is similar to material used by Wade [6] for obtaining near correlation with the experimental results.

To understand the experimental workings of the material, different coupon compression tests were conducted by Wade [6]. A good correlation with the experimental results is necessary to accurately predict the element failure in LSDyna. A sinusoidal wave coupon, Fig (2), was simulated under axial compression load. A trigger equivalent of $45^{\circ}$ chamfer was modelled, which initiated a progressive stable crushing. MAT_ENHANCED_COMPOSITE_DAMAGE (Mat 54) incorporates some model specific material failure parameters. These include element deletion time step size, material strength reduction factor, fibre tensile strength reduction factor and fibre strength reduction after matrix failure. These 
parameters were configured, Fig (2), to obtain similar material failure mechanism as observed experimentally.
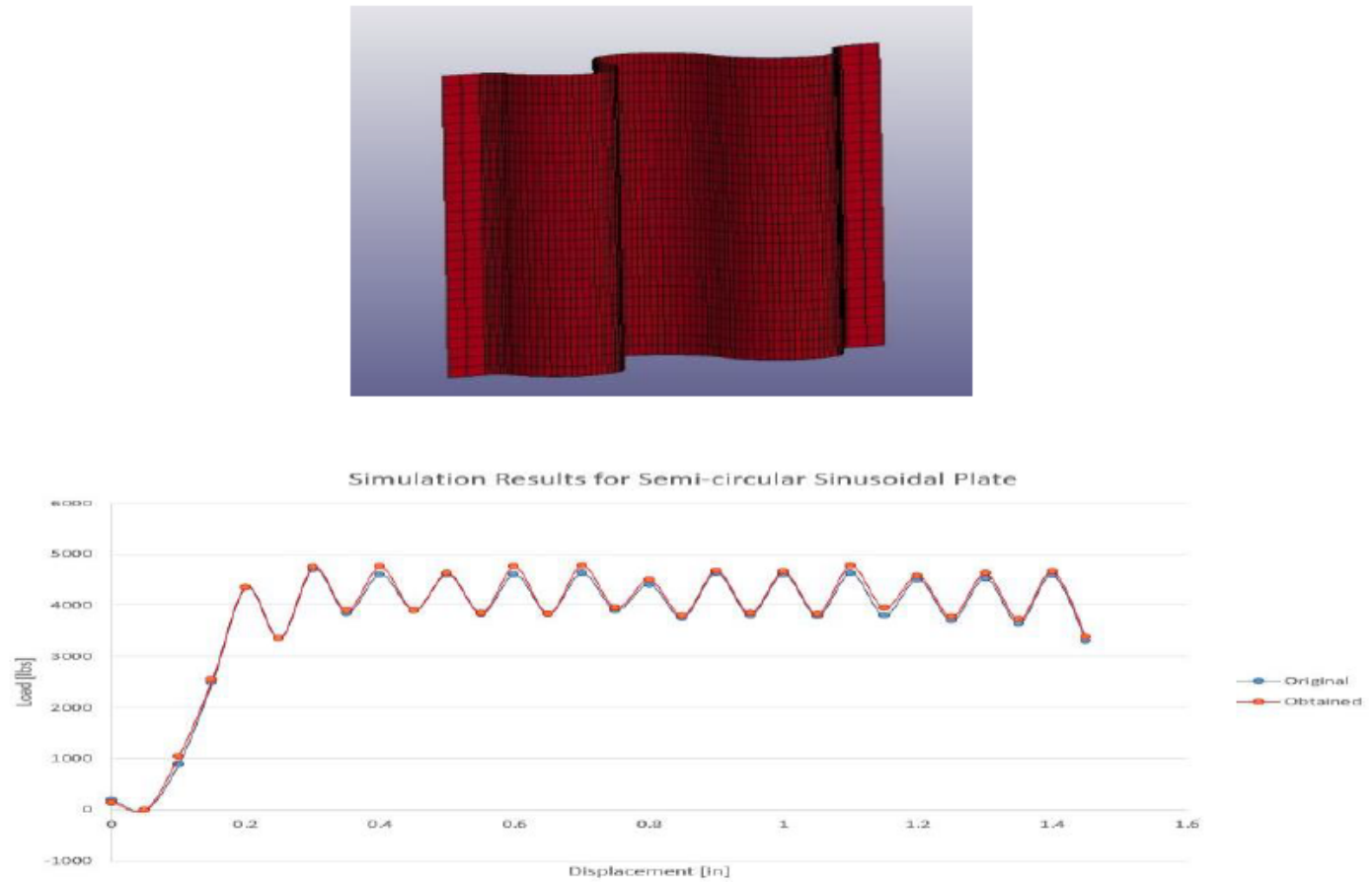

Figure 2. Sinusoidal coupon and correlation with experimental results.

From the material data sheet provided, the following properties were observed (Table I). The strains (Table II) for both fibre and matrix in tension as well as compression were calculated using the $\mathrm{ABD}$ stiffness matrix.

Table I. Material properties of T700/2510.

\begin{tabular}{|c|c|c|}
\hline Parameters & Values & Units \\
\hline Density ( $\rho)$ & $1.52 \mathrm{e}-6$ & $\mathrm{~kg} / \mathrm{mm}^{3}$ \\
\hline Young's Modulus along fiber $\left(E_{A}\right)$ & 127 & GPa \\
\hline Young's Modulus perpendicular to fiber $\left(E_{B}\right)$ & 8.41 & GPa \\
\hline Poisson's Ratio (PRBA) & 0.02 & - \\
\hline Fiber tensile strength (XT) & 22 & GPa \\
\hline Fiber compressive strength $(\mathrm{XC})$ & 14.7 & GPa \\
\hline Matrix tensile strength (YT) & 0.05 & GPa \\
\hline Matrix compressive strength (YC) & 0.2 & GPa \\
\hline Shear Strength (SC) & 0.154 & Gpa \\
\hline Shear Stress (G) & 4.21 & $\mathrm{GPa}$ \\
\hline
\end{tabular}




\begin{tabular}{|c|c|}
\multicolumn{1}{c|}{ Strains } & Values \\
\hline DFAILT & 0.0174 \\
\hline DFAILC & -0.0116 \\
\hline DFAILM & 0.024 \\
\hline DFAILS & 0.030 \\
\hline
\end{tabular}

\section{Sections}

The geometry of nosecone is divided into two parts- the cover and the cone. The cover is not included in the simulations as this part would be manufactured bonded separately with the crash structure. Also, since this part will not be cured with the rest of the structure it lacks the structural integrity and consistent manufacturing process to evaluate the effects on deceleration.

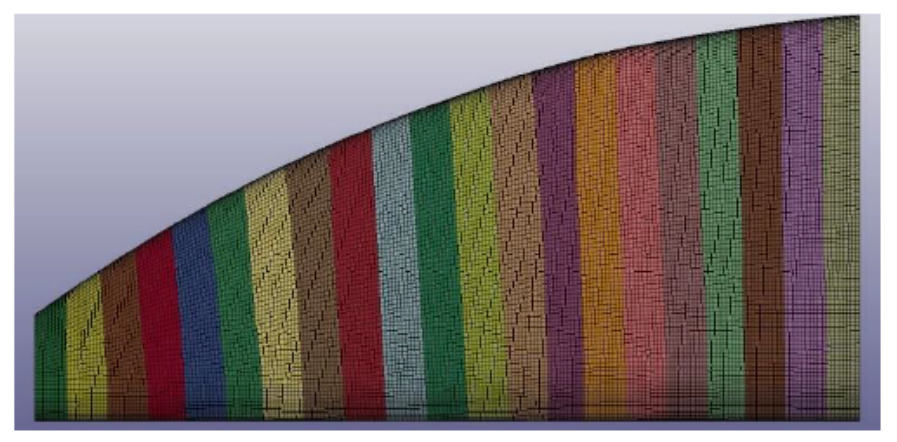

Figure 3. Longitudinal sections of nosecone.

The second part- cone is involved in the actual energy absorption of the nose cone consisting of a truncated pyramidal structure with a square section. The geometry is divided longitudinally into 21 sections Fig (3). The number of sections were considered based on manufacturing processes. Furthermore, the sections were divided only longitudinally as the research focuses on a $0^{\circ}$ angle of impact. Each section is individually defined to have a particular layup sequence, ply thickness and ply orientation. The design is completed with each section acting as a trigger for the proceeding section, thus leading to stable and controlled crushing as well as controlling the deceleration peaks. This methodology is intended for a dual application of reducing the value of the force peak and initializing structure collapse in a stable regime. 


\section{OPTIMIZATION}

A multilayered laminate is to be optimized for minimum mass subjected to fragmentation, splaying and global \& local buckling. The prepreg layups are of specific thickness, hence only number of layups and orientation of laminas are varied. To assess the two-dimensional failure of composite structures ChangChang's failure criterion was utilized. The optimization cost function is mass of laminate which can be represented as

$$
f=f\left(n, \theta_{i}\right)
$$

Where,

$\mathrm{n}=$ number of layups

$\theta_{i}=$ orientation of lamina

The initial layup for each section is calculated by utilizing the variation of SEA with $\varphi$, as suggested by Wade [6]. By comparing the computed deceleration of plate with the ideal deceleration curve, for a particular section, and the total Kinetic Energy absorbed by the section; the number of layups and layup sequence is varied to optimum values.

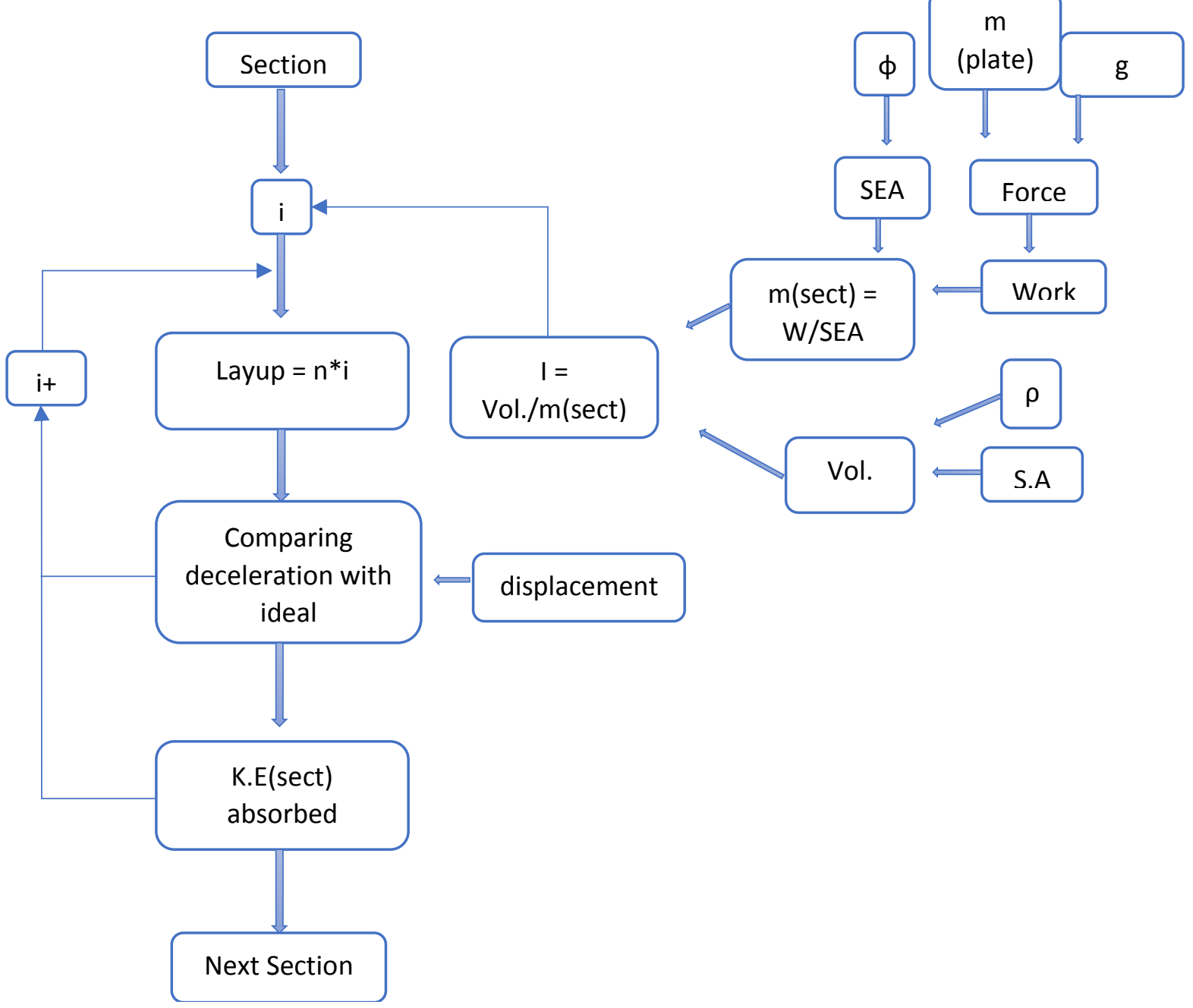

Figure 4. Optimization technique for each section of nosecone. 


\section{RESULTS \& DISCUSSIONS}

The resultant deceleration vs displacement curve, Fig (5), shows that in the first $150 \mathrm{~mm}$ of crushing, the deceleration was stabilized to $0.04 \mathrm{~mm} / \mathrm{ms}^{2}$ or $4 \mathrm{~g}$. An average deceleration of $12.45 \mathrm{~g}$ was observed, which is within the $25 \mathrm{~g}$ limit imposed by the rules. As there are no sudden dips or increases in the forces exerted on the structure, it can be concluded that the deformable structure collapses continuously. It was observed that an initial kinetic energy of $58.8 \mathrm{~kJ}$ was completed dissipated into splaying/fragmentation of fibers and into heat energy generated due to friction between the surfaces. The methodology of varying the number of plies with displacement, resulted in an optimized crash structure which met the rules and regulations imposed by FIA. The resultant weight of optimized structure was 2.1 kgs which is $41 \%$ less and improved weight than the conventional CFRPAluminium sandwich structures.

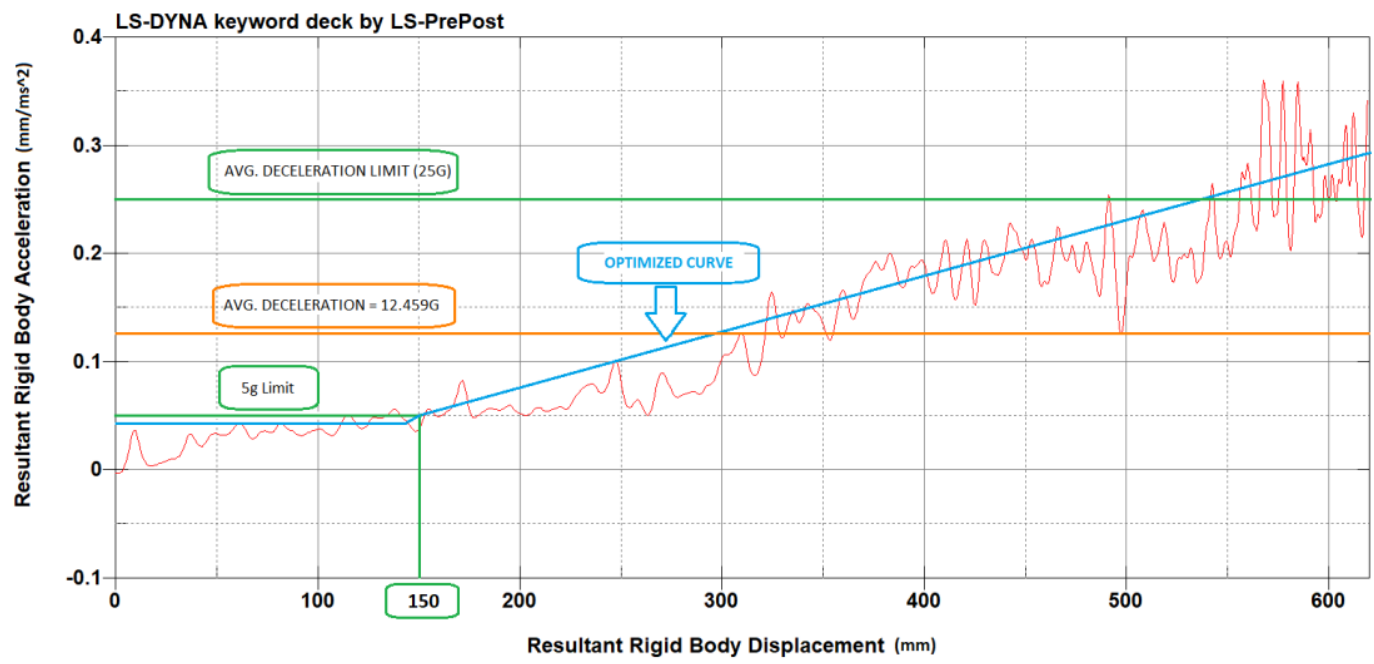

Figure 5. Resultant optimized deceleration vs displacement graph.

By dividing the crash structure into various sections, each having unique stacking sequence, the reactive forces acting on the structure can be controlled. By utilizing the variations in SEA for a particular cross-section, the energy dissipated per section can be controlled. Furthermore, the variation of SEA with the percentage of curvature $(\varphi)$ partially quantifies the energy absorption capacity of a structure with the geometry. (Table III) indicates the difference in calculated number of layers and the observed number of layers. As the angle of attack $(\beta)$ is increased the error difference in calculated layup increases. This can be contributed to the decrease in fragmentation and splaying of the material. With an increase in $\beta$, the fibers are subjected to bending rather than compression. Due to the absence of fibers or Aluminium core in the z-direction, there is little resistance to bending. If the $\beta$ is low, failure by fragmentation and splaying (both attributed to micro buckling) is predominant. This utilizes the compressive strength of fibers more effectively. However, if $\beta=0$ and no trigger is introduced, matrix shear is predominant in which case the fibers provide little resistance. Hence a stable 
crushing is not observed. To utilize the materials true potential, $\beta$ should be low and trigger should initiate stable progressive crushing.

Table III. Sectional layup and angle of attack.

\begin{tabular}{|c|c|c|c|c|}
\hline SECTION & INITIAL LAYUP & FINAL LAYUP & $\Delta$ & $\begin{array}{c}\text { Angle of attack } \\
(\beta)\end{array}$ \\
\hline 1 & 2 & 3 & 1 & 32.5 \\
\hline 2 & 3 & 5 & 2 & 30.5 \\
\hline 3 & 5 & 8 & 3 & 28.3 \\
\hline 4 & 5 & 8 & 3 & 25.8 \\
\hline 5 & 6 & 8 & 2 & 23.3 \\
\hline$\overline{6}$ & 7 & 9 & 2 & 21.2 \\
\hline 7 & 8 & 10 & 2 & 19 \\
\hline 8 & 9 & 11 & 2 & 17 \\
\hline 9 & 11 & 12 & 1 & 14 \\
\hline 10 & 12 & 13 & 1 & 12.8 \\
\hline 11 & 13 & 14 & 1 & 11.6 \\
\hline 12 & 14 & 15 & 1 & 10.5 \\
\hline 13 & 15 & 16 & 1 & 9.6 \\
\hline 14 & 16 & 17 & 1 & 8.9 \\
\hline 15 & 17 & 18 & 1 & 8.3 \\
\hline 16 & 18 & 19 & 1 & 7.9 \\
\hline 17 & 19 & 20 & 1 & 7.2 \\
\hline 18 & 20 & 21 & 1 & 6.8 \\
\hline 19 & 22 & 22 & 0 & 6.3 \\
\hline 20 & 22 & 22 & 0 & 5.7 \\
\hline 21 & 24 & 24 & 0 & 5.5 \\
\hline
\end{tabular}

\section{CONCLUSION}

The goal of this research was to utilize the SEA variation with percentage of curvature $(\varphi)$ for a particular geometry and optimize the structure for minimum weight. While the SEA calculations lead to a particular stacking sequence, the previous research failed to consider the angle of attack $(\beta)$, angle of impact and its impact on energy absorption capacity. As the geometry considered had an extreme angle of attack, the calculations failed to accurately represent the correct SEA. However, the sections with negligible $\beta$ had better estimations of SEA and could approximately predict the right ply sequence. Furthermore, as the angle of attack increased the error in ply sequence increased hence indicating a direct relation between SEA and angle of attack. 


\section{REFERENCES}

[1] Weaver, Christopher \& K Sloan, Brian \& J Brizendine, Edward \& Bock, Henry. "An Analysis of Maximum Vehicle G Forces and Brain Injury in Motorsports Crashes" Medicine and science in sports and exercise. 38. 246-9. 10.1249/01.mss.0000184773.07870.5e.

[2] FIA F3 Technical Regulations Appendix J Article 275-2013.

[3] Hull. D., "A unified approach to progressive crushing of fibre reinforced composite tubes", Composites Science and Technology, vol. 40, pp. 377-421, January 1991.

[4] Farley, G.L. and Jones, R.M. 1992. "Crushing Characteristics of Continuous Fiber-Reinforced Composite Tubes," Journal of Composite Materials, 26, pp. 37-50.

[5] Carruthers, J.J., Kettle, A.P., and Robinson, A.M. 1998. "Energy Absorption Capability and Crashworthiness of Composite Material Structures: A Review," Applied Mechanics Review, 51(10), pp. 635-649.

[6] Wade B., "Capturing the Energy Absorbing Mechanisms of Composite Structures under Crash Loading" University of Washington Dissertation 2014.

[7] Heimbs, Sebastian \& Strobl, F \& Middendorf, Peter \& Gardner, S \& Eddington, B \& Key, J. (2009). Crash Simulation of an F1 Racing Car Front Impact Structure.

[8] Feraboli. P., Wade. B., "Crushing Behavior of Laminated Composite Structural Elements: Experiment And LS-DYNA Simulation", DOT/FAA/TC-15/25, Federal Aviation Administration, Washington, DC, December 2016.

[9] Anghileri. M., Benetton. D., Bassi. S.M., "Numerical Modelling of a Crashabsorbing Nosecone", Matr. 876746, Dipartimento di Scienze e Tecnologie Aerospaziali, Politecnico di Milano, June 2018.

[10] Belingardi. G., Obradovic. J., Boria.S., "Experimental and numerical investigation of the impact behavior of composite frontal crash structures" Composite Part b, vol. 79, pp. 20-27, September 2015. 